\title{
On the role of asymmetries in the reversal of the solar magnetic field
}

\author{
A. S. Brun ${ }^{1}$, M. L. Derosa ${ }^{2}$ and J. T. Hoeksema ${ }^{3}$ \\ ${ }^{1}$ Laboratoire AIM Paris-Saclay, CEA/Irfu Université Paris-Diderot CNRS/INSU, 91191 \\ Gif-sur-Yvette, France \\ email: sacha.brun@cea.fr \\ ${ }^{2}$ Lockheed Martin Solar and Astrophysics Laboratory, 3251 Hanover St. B/252, Palo Alto, \\ CA 94304, USA \\ ${ }^{3}$ W. W. Hansen Experimental Physics Laboratory, Stanford University, Stanford, CA 94305, \\ USA
}

\begin{abstract}
We study how the solar magnetic field evolves from antisymmetric (dipolar) to symmetric (quadrupolar) state during the course of its 11-yr cycle. We show that based on equatorial symmetries of the induction equation, flux transport solar mean field dynamo models excite mostly the antisymmetric (dipolar) family whereas a decomposition of the solar magnetic field data reveals that both families should be excited to similar amplitude levels. We propose an alternative solar dynamo solution based on North-South asymmetry of the meridional circulation to better reconcile models and observations.
\end{abstract}

Keywords. Sun, dynamo, magnetic reversals, 11yr cycle

\section{Introduction}

The Sun possesses cyclic magnetic activity with a mean period of 22-yr, involving global field reversal of the poloidal field during the maximum phase of its 11-yr sunspot cycle. During such a reversal phase the Sun exhibits a much more complex field topology than during minimum where it is predominantly (but not exclusively as we will see below) dipolar. Understanding how magnetic fields reverse as part of the global dynamo process operating in the Sun is a very important element in our path toward a better and more realistic description of our star. The Sun is one of many celestial bodies that possess magnetic activity and a dynamo. In our solar system, for instance, the Earth also displays magnetic activity and global field reversals. Comparing both systems is likely to provide insight on the underlying dynamo process.

Indeed a recent analysis of geomagnetic records has indicated that the interplay between low-degree harmonic modes during polarity reversals is one way to characterize both reversals of the geomagnetic dynamo (which are irregular, but have a mean period of about 300,000 yr) as well as excursions, where the dipole axis temporarily moves equatorward and thus away from its usual position of being approximately aligned with the rotation axis, followed by a return to its original position without having crossed the equator (see Hulot et al. 2010 for a recent review on Earth's magnetic field). Such studies have shown that, during periods of geomagnetic reversals, the quadrupolar component of the geomagnetic field is stronger than the dipolar component, while during an excursion (which can be thought of as a failed reversal), the dipole remains dominant (Leonhardt \& Fabian 2007). One may thus ask: Is a similar behavior observed for the solar magnetic field? 
In an attempt to address this question, we have performed in Derosa, Brun \& Hoeksema (2012, hereafter DBH12), a systematic study of the temporal evolution of the solar photospheric field by determining the spherical harmonic coefficients for the photospheric magnetic field throughout the past three sunspot cycles, focusing on low-degree modes and the relative amplitudes of the dipolar and quadrupolar components. Following the classification of McFadden et al. (1991), we have also made the distinction between primary $(\ell+m$ odd $)$ and secondary $(\ell+m$ even $)$ families of harmonic modes, a classification scheme that takes into account the symmetry and parity of the spherical harmonic functions (see Gubbins \& Zhang (1993) for a detailed discussion on symmetry and dynamo as well as the Appendix B of DBH12).

While we recognize that the solar dynamo operates in a more turbulent parameter regime than the geodynamo, and is more regular in its reversals, the presence of grand minima (such as the Maunder Minimum) in the historical record indicates that the solar dynamo can switch to a more intermittent state on longer-term, secular time scales. In fact in the late stages of the Maunder Minimum, the solar dynamo was apparently asymmetric, with the southern hemisphere possessing more activity than the north (Ribes \& Nesme-Ribes 1993) for several decades, a magnetic configuration that may have been achieved by having dipolar and quadrupolar modes of similar amplitude (Tobias 1997, Gallet \& Petrelis 2009).

We here summarize our findings published in DBH12 and focus on mean field dynamo models and whether they reproduce the relative amplitude and phase of the axisymmetric modes with a special emphasis on the dipole and quadrupole.

\section{Observations of the solar magnetic field}

We analyze time series of synoptic photospheric magnetic field maps of the radial magnetic field $B_{r}$ derived from line-of-sight magnetogram observations taken by both the Wilcox Solar Observatory (WSO; Scherrer et al. 1977) at Stanford University and by the Michelson Doppler Imager (MDI; Scherrer et al. 1995) on board the space-borne Solar and Heliospheric Observatory (SOHO). The WSO data† used in this study span the past 36 years, commencing with Carrington Rotation (CR) 1642 (which began on 1976 May 27) and ending with CR 2123 (which ended on 2012 May 25). For MDI we used data from much of its mission lifetime, starting with CR 1910 (which began on 1996 Jul. 1) through CR 2104 (which ended on 2010 Dec. 24). In both data series, one map per Carrington rotation was used, though maps with significant amounts of missing data were excluded. The measured line-of-sight component of the field is assumed to be the consequence of a purely radial magnetic field when calculating the harmonic coefficients. Additionally, for WSO, the synoptic map data are known to be a factor of about 1.8 too low due to the saturation of the instrument (Svalgaard, Duvall \& Scherrer 1978). Lastly, the MDI data have had corrections applied for the polar fields using the interpolation scheme presented in Sun et al. (2011).

For each map, we perform the harmonic analysis using the Legendre-transform software provided by the "PFSS" package available through SolarSoft. Using this software first entails remapping the latitudinal dimension of the input data from the sine-latitude format provided by the observatories onto a Gauss-Legendre grid (c.f., $\S 25.4 .29$ of Abramowitz \& Stegun 1972). This regridding enables Gaussian quadrature to be used when evaluating the sums needed to project the magnetic maps onto the spherical harmonic functions. The end result is a time-varying set of complex coefficients $B_{\ell}^{m}(t)$ for a series of modes

$\dagger$ Available at http://wso.stanford.edu/synopticl.html.

$\ddagger$ Available at http://soi.stanford.edu/magnetic/index6.html. 


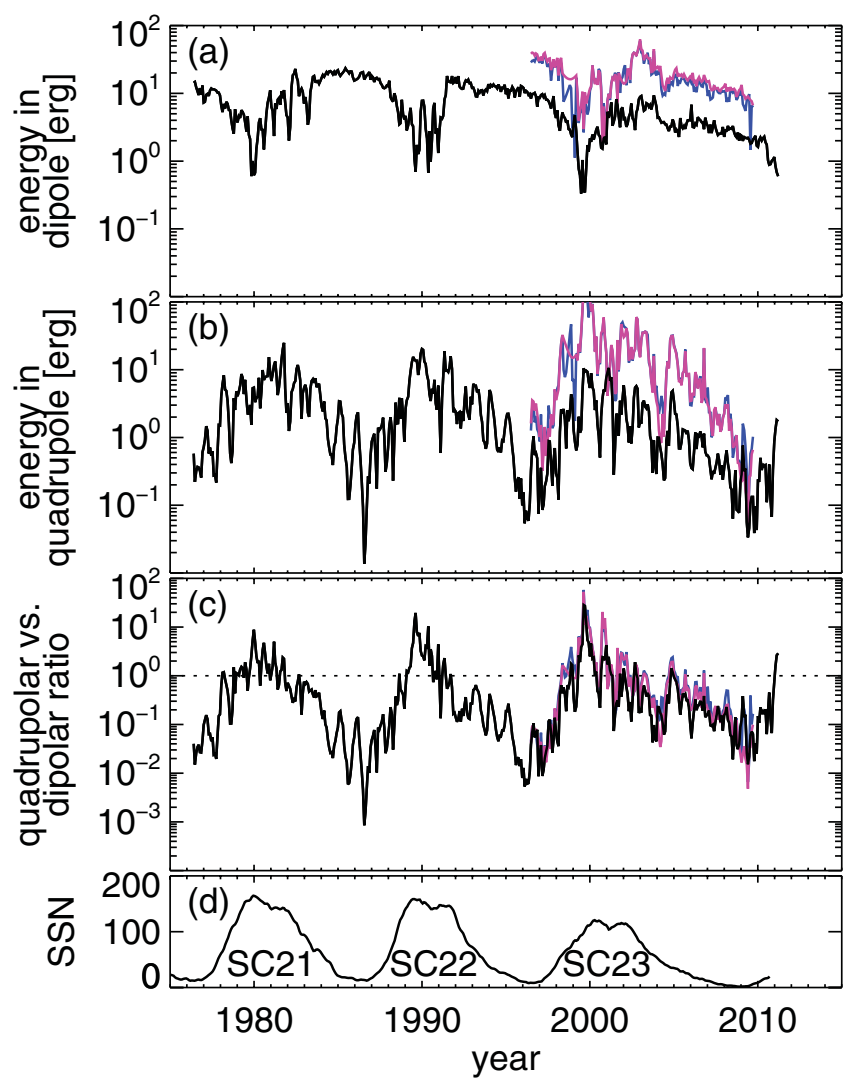

Figure 1. Total energy in (a) dipolar modes $\sum_{m}\left(B_{1}^{m}\right)^{2}$, (b) quadrupolar modes $\sum_{m}\left(B_{2}^{m}\right)^{2}$, and (c) their ratio $\sum_{m}\left(B_{2}^{m}\right)^{2} / \sum_{m}\left(B_{1}^{m}\right)^{2}$ for the WSO (dark tone) and MDI polar-corrected (light tone) data sets. Panel (d) shows the monthly smoothed SSN. The WSO data have not been corrected for known saturation effects that reduce the reported values by a factor of 1.8 Svalgaard et al. (1978) to provide an offset in the plots for visibility.

spanning harmonic degrees $\ell=0,1, \ldots, \ell_{\max }$, where the truncation limit $\ell_{\max }$ is equal to 60 for the WSO maps and 192 for MDI maps. The $B_{\ell}^{m}$ coefficients are proportional to the amplitude of each spherical harmonic mode $Y_{\ell}^{m}$ for degree $\ell$ and order $m$ possessed by the time series of synoptic maps, so that

$$
B_{r}(\theta, \phi, t)=\sum_{\ell=0}^{\ell_{\text {max }}} \sum_{m=0}^{\ell} B_{\ell}^{m}(t) Y_{\ell}^{m}(\theta, \phi),
$$

where $\theta$ is the colatitude, $\phi$ is the latitude, and $t$ is time. We note that because the coefficients $B_{\ell}^{m}$ are complex numbers, this naturally accounts for the rotational symmetry between spherical harmonic modes with orders $m$ and $-m$ (for a given value of $\ell$ ), with the amplitudes of modes for which $m>0$ appearing in the real part of $B_{\ell}^{m}$, and the amplitudes of the modes where $m<0$ being contained in the imaginary part of $B_{\ell}^{m}$. Consequently, the sum over $m$ in equation (2.1) starts at $m=0$ instead of at $m=-\ell$. The coefficients $B_{\ell}^{0}$ corresponding to the axisymmetric modes (for which $m=0$ ) are real for all $\ell$.

In Figure 1 we display the squares of the amplitudes of the dipolar and quadrupolar modes (having summed over all $m$ 's) over the last 3 cycles. We also show in panel (c), 


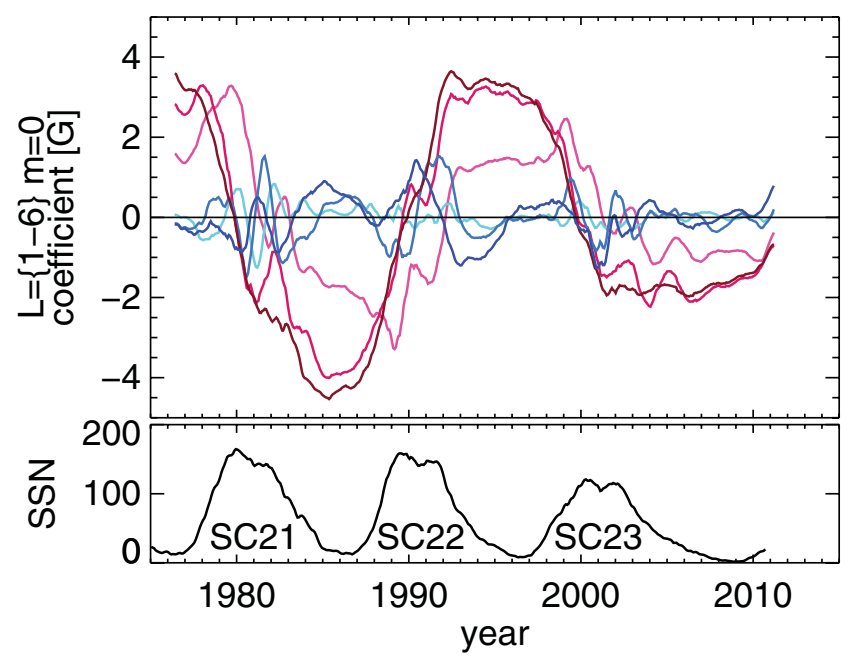

Figure 2. Overplotted coefficients $B_{\ell}^{0}$ of the first 3 odd $(\ell=\{1,3,5\}$; dark red, red, light red lines, respectively; or higher amplitude curves in BW version) and even $(\ell=\{2,4,6\}$; dark blue, blue, light blue, respectively; or lower amplitude curves in BW version) axisymmetric modes, as calculated from WSO synoptic maps.

their ratio. It is immediately evident that the quadrupole and dipole are in quadrature of phase, with the dipole being dominant most of the time except near solar cycle maxima. We note that, as for the Earth, a reversal implies that modes of the secondary family (here the $l=2, m=0$ and $(\ell=1, m=1)$ are dominant. However to our knowledge no failed reversals (e.g. excursions) have been recorded for the Sun contrary to the Earth.

In Figure 2 we display the first six axisymmetric $m=0$ low- $\ell$ modes. We note that the modes of the secondary family (here in the axisymmetric case the even $\ell$ 's) are weaker than the modes of the primary family. However even over the quiet (minimum) phase of the cycle, they are not negligible (of the order of $25 \%$ ), indicating that the Sun excites both families. This is very important as this shows that field reversals are intimately linked to the coupling of both families of mode in particular the axisymmetric dipole and quadrupoles.

\section{Mean field solar dynamo models: the role of flow asymmetry}

In order to confront the solar observational data with our current understanding of the solar dynamo and 22-yr cycle we have computed mean field solar dynamo models using the STELEM code (Emonet \& Charbonneau 1998 private communication, Jouve \& Brun 2007). With the current classical ingredients (meridional circulation, surface source term) used in flux transport dynamo models it is indeed possible to reproduce a 22-yr cycle. We display in Figure 3 (left panel) the butterfly diagram (e.g a time-latitude plot of the toroidal and radial magnetic field) obtained with such models. While a clear equatorward branch is seen as with the Sun, we note the predominantly dipolar (antisymmetric with respect to the equator) pattern. A decomposition on Legendre polynomials of this model indicates that the secondary family modes are more than 5 orders of magnitude smaller than the modes of the primary family. We further note that both hemispheres are in phase, reversing at the same time.

We know from symmetry considerations of the induction equation (GZ93, DBH12) that kinematic dynamo with purely symmetric flows and an antisymmetric poloidal source 

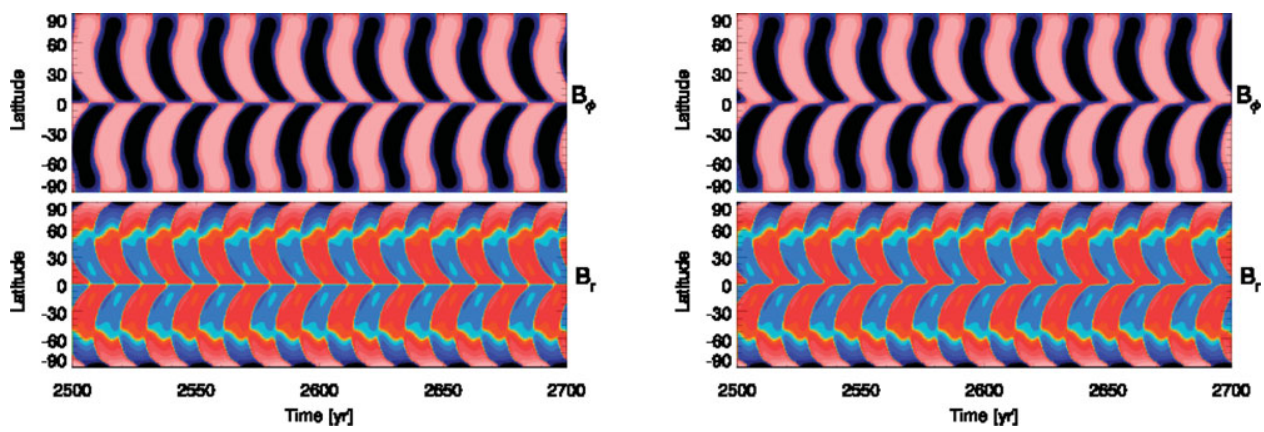

Figure 3. Magnetic butterfly diagram (time-latitude plot of the magnetic field). a) Standard case (JB2007). b) North-South asymmetric case (DBH12).

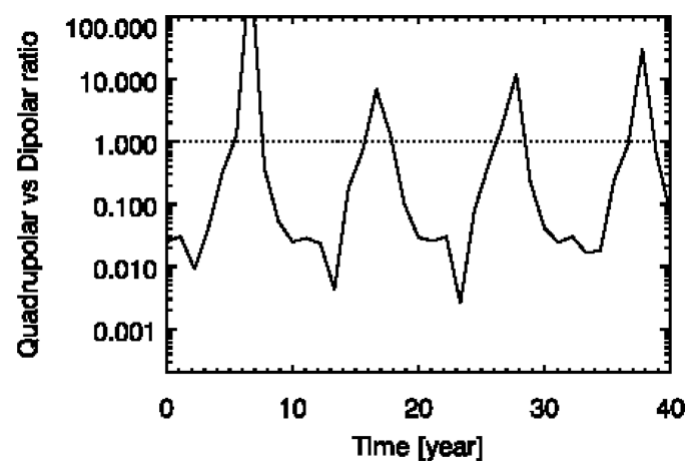

Figure 4. Ratio of the axisymmetric dipole and quadrupole coefficient in the asymmetric mean field dynamo case shown in Fig 3b (right). Note how the ratio follows qualitatively well the observations shown in Fig1 panel (c).

term do not couple both dynamo families. Nonlinearities as in 3-D simulations (Brun et al. 2004) or in 2-D nonlinear dynamo (Tobias 1997, 2002) allows for such coupling. Another possibility is to consider flows or field source asymmetric with respect to the equator. We have thus modified the standard mean field flux transport dynamo model to take into account such asymmetries of the main physical ingredients (meridional flow, surface source term) and computed several models by varying the degree of asymmetry $\epsilon$. On Figure 3 (right panel) we display a solar mean field model where the meridional circulation possesses a degree of asymmetry $\epsilon$ of $10^{-3}$. We see that while the butterfly diagram still exhibits dipolar (antisymmetric) magnetic field it is not strictly antisymmetric hinting to the presence of symmetric (quadrupolar) modes. To illustrate the role played by the secondary family modes, we plot in Figure 4 the ratio between the quadrupolar and the dipolar axisymmetric modes. With an asymmetric parameter $\epsilon$ set to $10^{-3}$ we indeed see that this reproduces qualitatively well the solar observations shown on Fig 1 (panel c), with a ratio varying between $10^{-3}$ and $10^{2}$. We also note that the reversal in each hemisphere does not occur exactly in phase. Actually with such a degree of asymmetry we find that the North pole reverses more than $1 \mathrm{yr}$ ahead of the South pole. This is compatible with solar observations, that clearly show that one hemisphere can be ahead of the other one by up to $2 \mathrm{yr}$ (Dikpati et al. 2007, Shiota et al. 2012, Tsuneta et al. 2012 this proceedings). Here we did not tune the amplitude nor the sign of the asymmetry parameter $\epsilon$ to reproduce the sequence of reversals and time lag between the Northern and Southern hemispheres seen in the Sun but we intend to do so in the near future. 


\section{Discussion}

We have shown that the Sun excites both dynamo families, wherein during field reversals the quadrupolar-like (secondary) modes become dominant as seen in the geomagnetic records, and that the low- $\ell$ secondary modes remain of significant amplitude even during more quiescent phases (e.g. away from the maximum of activity). From our analysis of MDI and Wilcox magnetogram data we have looked in particular at the axisymmetric modes from $\ell=1$ to 6 , since these are the ones most easily compared with the output from 2-D mean field dynamo models. When comparing standard flux transport dynamo models (Jouve \& Brun 2007, Charbonneau 2010, Choudhuri 2012 (this proceedings)) with solar observations it was noticed that in such models the secondary family is not excited and does not maintain a level similar to that seen on the Sun (DBH12). This is expected from the equatorial symmetry of the flow profiles and poloidal source terms used in most mean field dynamo models (see GZ93, McFadden et al. 2011, DBH12). We have shown that by modifying the ingredients of the standard dynamo models by introducing a certain degree of asymmetry $\epsilon$ in the flow both families are coupled more efficiently. We find that we can qualitatively reproduce the ratio between the quadrupole and the dipole observed in the Sun and also explain the origin of the time lag seen in the reversal of the magnetic field between the North and the South poles (Dikpati et al. 2007, Shiota et al. 2012, Tsuneta et al. 2012 (this proceedings)).

\section{References}

Abramowitz, M. \& Stegun, I. A., eds. 1972, Handbook of Mathematical Functions with Formulas, Graphs, and Mathematical Tables (New York: Dover)

Brun, A. S., Miesch, M. S., \& Toomre, J. 2004, ApJ, 614, 1073

Charbonneau, P. 2010, Living Rev. Sol. Phys., 7, 3

DeRosa, M. L., Brun, A. S., \& Hoeksema, J. T. 2012, ApJ, 757, 96 (DBH12)

Dikpati, M., Gilman, G. A., de Toma, G., \& Ghosh, S. S. 2007, Sol. Phys., 245, 1

Gallet, B. \& Pétrélis, F. 2009, Phys. Rev. E, 80, 035302

Gubbins, D. \& Zhang, K. 1993, Phys. Earth Planet. Inter., 75, 225 (GZ93)

Hulot, G., Finlay, C. C., Constable, C. G., Olsen, N., \& Mandea, M. 2010, Space Sci. Rev., 152, 159

Jouve, L. \& Brun, A. S. 2007, A\&BA, 474, 239

Leonhardt, R. \& Fabian, K. 2007, Earth Planet. Sci. Lett., 253, 172

McFadden, P. L., Merrill, R. T., McElhinny, M. W., \& Lee, S. 1991, J. Geophys. Res., 96, 3923

Ribes, J. C. \& Nesme-Ribes, E. 1993, A\&A, 276, 549

Scherrer, P. H., Wilcox, J. M., Svalgaard, L., Duvall, Jr., T. L., Dittmer, P. H., \& Gustafson, E. K. 1977, Sol. Phys., 54, 353

Scherrer, P. H., Bogart, R. S., Bush, R. I., Hoeksema, J. T., Kosovichev, A. G., Schou, J., Rosenberg, W., Springer, L., Tarbell, T. D., Title, A., Wolfson, C. J., \& Zayer, I., MDI Engineering Team. 1995, Sol. Phys., 162, 129

Shiota, D., Tsuneta, S., Shimojo, M., et al. 2012, ApJ, 753, 157

Sun, X., Liu, Y., Hoeksema, J. T., Hayashi, K., \& Zhao, X. 2011, Sol. Phys., 270, 9

Svalgaard, L., Duvall, Jr., T. L., \& Scherrer, P. H. 1978, Sol. Phys., 58, 225

Tobias, S. M. 1997, A\& A, 322, 1007

-. 2002, Astron. Nachr., 323, 417 